\title{
Há evidências científicas na medicina antienvelhecimento?
}

\author{
Is there any scientific evidence supporting antiaging medicine?
}

Milton Luiz Gorzoni ${ }^{1}$

\author{
Sueli Luciano Pires ${ }^{2}$
}

\begin{abstract}
Resumo: A medicina antienvelhecimento visa a interferir no processo de envelhecimento humano biológico normal. Haveria base científica para justificá-la como especialidade médica e não ramo de ciências biológicas básicas? Esta revisão avaliou 110 artigos, dos quais nove artigos (8,2\% do total) em humanos. Apenas um desses estudos era randomizado e duplo-cego (Jadad 2). A categoria de recomendação e o grau de evidência desses trabalhos foram considerados como CII. Periódicos com fator de impacto acima de 1,110 publicaram três dos nove artigos. Dessa forma, não parece haver sólidas evidências científicas e/ou clínicas que justifiquem a aplicabilidade da medicina antienvelhecimento na presente prática médica.

Palavra-chave: Envelhecimento; Medicina baseada em evidências; Medicina clínica
\end{abstract}

\begin{abstract}
The objective of antiaging medicine is to interfere in the normal human biological aging process. Is there any scientific basis to justify classifying antiaging medicine as a medical specialty and not a branch of basic biological science? This review evaluated 110 papers, nine of which $(8.2 \%$ of the total) reported studies involving human subjects. Only one of these studies was randomized and double-blinded (Jadad 2). In accordance with their classification of recommendations and level of evidence, these studies were considered CII. Three of the nine articles were published in journals with an impact factor over 1.110. Therefore, there does not appear to be any solid scientific and/or clinical evidence that would justify the application of antiaging medicine in current medical practice.

Keywords: Aging; Clinical medicine; Evidence-based medicine
\end{abstract}

\section{INTRODUÇÃO}

O princípio básico da medicina antienvelhecimento - retardar, parar ou reverter o processo de envelhecimento humano biológico normal - contrapõe-se à biogerontologia, que distingue entre o envelhecer como um fenômeno natural e o papel do envelhecimento como fator de risco para determinadas doenças. Devem-se também distinguir condutas de seus praticantes dos atos relacionados com a saúde pública quanto à promoção de saúde e prevenção de doenças.

Órgãos e associações de classe, convênios médicos ou seguros-saúde não reconhecem, no Brasil, medicina antienvelhecimento como especialidade médica. Seu campo de ação abrange basicamente: ${ }^{1}$ a. Redução do estresse oxidativo por meio de suplementos dietéticos e/ou minerais e/ou vitamínicos; b. Restrição calórica e/ou uso de pré ou probióticos; c. Reposição e/ou suplementação hormonal;

d. Fitoterápicos;

e. Atividade física.

Pergunta-se, assim: qual seria sua base e/ou evidências científicas que justificariam sua existência como especialidade de medicina humana e não ramo de ciências biológicas básicas?

Este artigo de revisão visa a analisar, utilizando padrões de metodologia científica baseados em evidências, estudos publicados em periódicos da área da saúde humana que abranjam técnicas, procedimentos

\footnotetext{
Aprovado pelo Conselho Editorial e aceito para publicação em 17.04.2009.

* Trabalho desenvolvido no Hospital Geriátrico e de Convalescentes Dom Pedro II e disciplina "Fundamentos de Gerontologia" do Departamento de Clínica Médica da Faculdade de Ciências Médicas da Santa Casa de São Paulo - São Paulo (SP), Brasil.

O presente trabalho faz parte do Projeto n. 371/08, aprovado pelo Comitê de Ética em Pesquisa da Irmandade da Santa Casa de Misericórdia de São Paulo Conflito de interesse: Nenhum / Conflict of interest: None

Suporte financeiro: Nenhum / Financial funding: None

1 Professor adjunto - Hospital Geriátrico e de Convalescentes Dom Pedro II e disciplina "Fundamentos de Gerontologia" do Departamento de Clínica Médica da Faculdade de Ciências Médicas da Santa Casa de São Paulo - São Paulo (SP), Brasil.

Professora instrutora - Hospital Geriátrico e de Convalescentes Dom Pedro II e disciplina "Fundamentos de Gerontologia" do Departamento de Clínica Médica da Faculdade de Ciências Médicas da Santa Casa de São Paulo - São Paulo (SP), Brasil.

(C) 2010 by Anais Brasileiros de Dermatologia
} 
e terapias antienvelhecimento, além de procurar definir o grau de evidência e o percentual desses estudos publicados que possuem sólida estrutura metodológica e aplicabilidade na presente prática clínica.

Para a elaboração deste artigo, acessou-se o portal http://www.nlm.nih.gov (MediLine/PubMed) em 30 de agosto de 2008, pesquisando artigos pelos unitermos antiaging medicine. Excluíram-se trabalhos relacionados a revisões, editoriais ou discussões; descrições sobre técnicas e procedimentos cosméticos ou laboratoriais e trabalhos em células ou em animais. Os estudos encontrados que apresentavam casuísticas humanas foram analisados preferencialmente pela sua versão eletrônica e/ou pelo seu resumo nesse portal ou no relativo ao periódico que o publicou. Essa análise baseou-se nos critérios descritos abaixo:

a. Pelo escore de Jadad et al. $^{2}$ (1996), que avalia as probabilidades de viés do estudo por sete itens:

1. Estudo descrito como randomizado ou com palavras correlatas?

2. Estudo descrito como duplo-cego?

3. Descrição de retirada de fármacos anteriores?

4. Descrição de método apropriado para sequência de randomização?

5. Descrição de método apropriado para material duplo-cego?

6. Método inadequado para randomização?

7. Método impróprio para duplo-cego?

As questões um a cinco são indicações de trabalho de boa qualidade e valem um ponto cada se "sim" e nenhum ponto se "não". Se as questões seis e sete forem afirmativas, deve-se subtrair um ponto relativo a cada uma delas na somatória final. Esse cálculo não julga todos os elementos do estudo, mas pode ser útil em avaliações qualitativas entre fraca (zero) e forte (cinco pontos).

b. Por uma versão sumarizada dos graus de recomendação e de evidência do Oxford Centre for Evidence based Medicine: ${ }^{3}$

\section{Categoria de recomendação}

A - Boa evidência para apoiar a recomendação

B - Moderada evidência para apoiar a recomendação

C - Pobre evidência para apoiar ou não a recomendação

\section{Grau de Evidência}

I - $\geq 1$ estudo controlado e randomizado corretamente

II $-\geq 1$ estudo clínico ou caso-controle ou observações

III - Experiência clínica, estudos descritivos ou relatórios c. Pelo fator de impacto em lista de periódicos científicos no Journal Citation Report - 2003 Science Edition. ${ }^{4}$

\section{RESULTADOS DA REVISÃO}

O portal http://www.nlm.nih.gov (Mediline/PubMed), em 30 de agosto de 2008, apresentou 110 artigos pelos unitermos antiaging medici$n e,{ }^{1,5-113}$ sendo 103 integralmente em inglês e sete em outros idiomas (cinco em chinês, um em japonês e outro em alemão), apenas com seus resumos em inglês. Seu conteúdo dividia-se em:

a. 55 artigos (50,0\% do total) relativos a revisões, editoriais e discussões. $1,6,9,11,112,21,24,27,29,34,36,39-48,50-56,58,60-61,63,68,72-76,78-$ 80,82,83,88,89,95-97,99,104,107,110,112 Esses artigos não apresentaram revisões sistemáticas, ou seja, conduzidas de acordo com critérios pré-definidos numa tentativa de limitar vieses dos investigadores. Revisões sistemáticas com frequência incluem como meta análises dos dados dos estudos avaliados nelas, também não observadas nesses artigos.

b. Um artigo sobre princípios gerais da medicina antienvelhecimento ${ }^{7}$ e cinco sobre técnicas e procedimentos cosméticos ou laboratoriais. ${ }^{8,32-33,59,90}$

c. Um artigo não pôde ser avaliado devido a não ter nem seu resumo accessível por via eletrônica. 66 Outro não apresentava relação com medicina antienvelhecimento. $^{13}$

d. Os restantes 47 estudos abrangiam principalmente fitoterápicos e trabalhos em células, animais (de insetos a primatas) e humanos.

Nessa última categoria - seres humanos encontraram-se nove artigos ${ }^{14,17,25,28,30,64,67,86,109}(8,2 \%$ do total), sendo que suas principais características estão sumarizadas na Tabela 1. Há apenas um desses trabalhos $^{109}$ com descrição de estudo randomizado e duplocego (Jadad 2) e outros três ${ }^{25,30,86}$ com grupo controle. Observaram-se oito estudos clínicos ou observacionais ${ }^{14,17,25,28,30,64,67,86}$ e análise estatística adequadamente descrita em três desses artigos. ${ }^{25,28,67}$

Cada um dos nove trabalhos direcionou-se para técnicas, procedimentos e terapias diferentes, sendo envelhecimento cutâneo o único fator correlato a quatro desses estudos. ${ }^{14,28,30,67}$

A categoria de recomendação desses nove arti$\operatorname{gos}^{14,17,25,28,30,64,67,86,109}$ pode ser considerada "C", e o grau de evidência, "II". ${ }^{3}$

Apenas três dos periódicos que publicaram esses $\operatorname{artigos}^{28,30,109}$ apresentaram fator de impacto acima de 1,110, colocando-os na lista das 1.500 revistas científicas com melhores pontuações por esse critério. ${ }^{114}$ Por outro lado, dois outros desses periódicos não apresentaram fator de impacto. ${ }^{25,86}$ 
TABela 1: Principais características de artigos sobre medicina antienvelhecimento em casuísticas de seres humanos e indexados no portal MedLine/PubMed em 30/08/08

\begin{tabular}{|c|c|c|c|c|}
\hline $\begin{array}{l}\text { Autores - ano de } \\
\text { publicação } \\
\text { (avaliado pelo) }\end{array}$ & Casuística & Objetivo & Método & Resultado \\
\hline $\begin{array}{l}\text { Akhtar e Yazan }^{14}- \\
2008 \text { (resumo) }\end{array}$ & $\begin{array}{l}11 \text { voluntários } \\
4 \text { semanas }\end{array}$ & $\begin{array}{l}\text { Ação antienvelheci- } \\
\text { mento de emulsão } \\
\text { com vitamina C e pro- } \\
\text { teína de trigo }\end{array}$ & $\begin{array}{l}\text { Aplicação tópica de emulsão } \\
\text { com e sem vitamina C e pro- } \\
\text { teína de trigo. } \\
\text { Método estatístico não } \\
\text { descrito }\end{array}$ & $\begin{array}{l}\text { Sem diferenças } \\
\text { clínicas ou estatísticas }\end{array}$ \\
\hline $\begin{array}{l}\text { Benito-Ruiz et al. }{ }^{17}- \\
2008 \text { (artigo) }\end{array}$ & 21 homens & $\begin{array}{l}\text { Implante de músculo } \\
\text { peitoral para maior } \\
\text { volume torácico }\end{array}$ & $\begin{array}{l}\text { Cirúrgico } \\
\text { Método estatístico não descrito }\end{array}$ & $\begin{array}{l}\text { Resultados estetica- } \\
\text { mente satisfatórios }\end{array}$ \\
\hline $\begin{array}{l}\text { Shameem e Latif }{ }^{25}- \\
2007 \text { (resumo) }\end{array}$ & $\begin{array}{l}60 \text { idosos saudáveis } \\
\text { ( } \geq 60 \text { anos) e grupo } \\
\text { controle }-23 \text { adul- } \\
\text { tos jovens ( } 20 \text { a } 30 \\
\text { anos) }\end{array}$ & $\begin{array}{l}\text { Correlação de } \\
\text { envelhecimento com } \\
\text { concentração basal de } \\
\text { cortisol em idosos } \\
\text { saudáveis }\end{array}$ & $\begin{array}{l}\text { Radioimunoensaio } \\
\text { Análise estatística pelo pro- } \\
\text { grama SPSS usando teste t de } \\
\text { Student não pareado }\end{array}$ & $\begin{array}{l}\text { Sem diferenças } \\
\text { estatísticas entre os } \\
2 \text { grupos }\end{array}$ \\
\hline $\begin{array}{l}\text { Metcalf et al. }{ }^{28}- \\
2007 \text { (resumo) }\end{array}$ & $\begin{array}{l}26 \text { portadores de } \\
\text { lentigo maligna } \\
3 \text { meses }\end{array}$ & $\begin{array}{l}\text { Efetividade do creme } \\
\text { de imiquimode } 5,0 \% \\
\text { no fotoenvelhecimento }\end{array}$ & $\begin{array}{l}\text { Anatomopatológico de } \\
\text { biópsias cutâneas pré e } \\
\text { pós-tratamento. } \\
\text { Análise estatística - } \\
\text { teste Wilcoxan }\end{array}$ & $\begin{array}{l}\text { Melhora anatomo- } \\
\text { patológica estatisti- } \\
\text { camente significante }\end{array}$ \\
\hline $\begin{array}{l}\text { Fournier et al. }^{30}- \\
2006 \text { (resumo) }\end{array}$ & Não relatada & $\begin{array}{l}\text { Programa de rejuve- } \\
\text { nescimento cutâneo }\end{array}$ & $\begin{array}{l}\text { Grupo teste } \\
\mathbf{x} \\
\text { grupo controle. } \\
\text { Método estatístico não descrito }\end{array}$ & $\begin{array}{l}\text { Melhora clínica do } \\
\text { grupo teste }\end{array}$ \\
\hline $\begin{array}{l}\text { Yang et al. } .^{64}-2003 \\
\text { (resumo) }\end{array}$ & $\begin{array}{l}36 \text { homens com } \\
\text { prostatite crônica }\end{array}$ & $\begin{array}{l}\text { Ação de fitoterápico } \\
\text { em sêmen humano } \\
\text { Ação tópica de } \\
\text { fitoterápico em rugas }\end{array}$ & $\begin{array}{l}\text { Análise do sêmen antes e } \\
\text { após } 60 \text { dias de tratamento. } \\
\text { Métodos laboratoriais descritos. } \\
\text { Análise estatística }\end{array}$ & $\begin{array}{l}\text { Melhora com signifi- } \\
\text { cância estatística da } \\
\text { qualidade seminal }\end{array}$ \\
\hline $\begin{array}{l}\text { Bauza et al. }{ }^{67}-2002 \\
\text { (resumo) }\end{array}$ & $\begin{array}{l}10 \text { mulheres } \\
\text { (46 a } 58 \text { anos) }\end{array}$ & $\begin{array}{l}\text { Modulação da } \\
\text { imunidade por }\end{array}$ & $\begin{array}{l}\text { Descrição do padrão de análi- } \\
\text { se em } 5 \text { semanas de aplicação. } \\
\text { Análise estatística - teste } \\
\text { Wilcoxan }\end{array}$ & $\begin{array}{l}\text { Melhora em } 6 \\
\text { mulheres }\end{array}$ \\
\hline $\begin{array}{l}\text { Liu et al.86 - } 2000 \\
\text { (resumo) }\end{array}$ & $\begin{array}{l}47 \text { idosos e } 15 \\
\text { adultos jovens }\end{array}$ & fitoterápico & $\begin{array}{l}\text { Descrição dos métodos labo- } \\
\text { ratoriais. } \\
\text { Análise estatística }\end{array}$ & $\begin{array}{l}\text { Significância estatís- } \\
\text { tica. Possível uso } \\
\text { clínico futuro }\end{array}$ \\
\hline $\begin{array}{l}\text { Predescu et al. }^{109}- \\
1994 \text { (resumo) }\end{array}$ & 343 idosos & $\begin{array}{l}\text { Fórmula } \\
\text { antienvelhecimento e } \\
\text { antiestresse em } \\
\text { psiquiatria }\end{array}$ & $\begin{array}{l}4 \text { grupos: (1) Meclofenoxate, } \\
\text { (2) Nicergolina, (3) Placebo, } \\
\text { (4) Fórmula. } \\
\text { Estudos duplo-cegos e rando- } \\
\text { mizados. } \\
\text { Escalas de avaliação padroni- } \\
\text { zadas e usuais. } \\
3 \text { meses de tratamento }\end{array}$ & $\begin{array}{l}\text { Não quantifica } \\
\text { resultados. } \\
\text { Os } 3 \text { grupos com } \\
\text { fármacos melhores } \\
\text { que o de placebo. } \\
\text { Discute mono x } \\
\text { multiterapia em } \\
\text { idosos }\end{array}$ \\
\hline
\end{tabular}




\section{COMENTÁRIOS SOBRE A REVISÃo}

Estudos laboratoriais, em células ou em animais são os primeiros passos para futuras pesquisas e terapias em seres humanos. Há, porém, distâncias significativas entre pesquisas com Drosopbila melanogaster $^{91}$ (mosca das frutas) ou Musca domestica ${ }^{84,91}$ (mosca comum) e sua aplicabilidade imediata em humanos. Esse seria o mais importante equívoco metodológico da medicina antienvelhecimento - a inferência, ou seja, transpor estudos de ciência básica para a prática médica sem passar pelas fases de pesquisa clínica.

Define-se como pesquisa clínica, também denominada ensaio ou estudo clínico, o processo de investigação científica que envolve seres humanos. Busca basicamente o desenvolvimento de novos medicamentos e procedimentos capazes de agir sobre a saúde de humanos. Sua execução exige o cumprimento de etapas contidas em protocolos para esse fim e com base em regulamentação nacional e internacional. Quando vinculada ao estudo de medicamentos, vacinas ou procedimentos diagnósticos, deve-se classificá-la em uma das quatro fases possíveis de investigação clínica: ${ }^{115-118}$

a. Fase 1 - medicamento utilizado pela primeira vez em humanos, normalmente saudáveis e não portadores da doença na qual o fármaco agiria. Avaliam-se diferentes vias de administração e doses, realizam-se testes iniciais de segurança e de interações medicamentosas. Habitualmente o número de participantes fica entre 20 e 100.

b. Fase 2 - conhecida também como "estudo piloto", essa fase abrange portadores da doença na qual o fármaco agiria, visando a determinar sua segurança e eficácia. As casuísticas oscilam entre 100 e 300 pacientes. c. Fase 3 - concluída a fase 2 com sucesso, aumentase o número de doentes nos denominados "estudos terapêuticos ampliados ou multicêntricos", com estrutura metodológica semelhante ao da fase anterior. São estudos de duração temporal maior e comparativos a placebo ou a tratamentos já existentes para a doença em análise. Seu objetivo básico é a obtenção de melhores informações, qualitativa e quantitativamente, sobre segurança, eficácia e interações do fármaco utilizado. Os resultados relativos a essa fase, se positi- vos, contribuem para o registro e aprovação em uso comercial do novo medicamento pelas autoridades sanitárias. Estudos dessa fase necessitam de 300 a 3.000 pacientes para sua execução.

d. Fase 4 - também conhecida como "estudo pós-marketing”, é realizada com o intuito de avaliar a eficácia e detectar efeitos colaterais do fármaco em populações que consomem o medicamento vendido livremente aos consumidores, que, nessa fase, são em número de milhares ou de milhões.

Excetuando-se o trabalho de Predescu et al. com 343 idosos, os outros oito estudos ${ }^{14,17,25,28,30,64,67,86}$ relataram casuísticas entre 10 e 83 humanos, ou seja, número de participantes compatível com pesquisas clínicas da fase 1. Apenas dois estudos ${ }^{28,64}$ utilizaram fármacos em portadores de doença, mas não apresentaram número de pacientes para serem classificados como fase 2 . Igualmente a descrição do estudo como randomizado e duplo-cego (Jadad 2) ocorreu em um único trabalho, ${ }^{109}$ e o cuidado de utilizar grupo controle para efeito comparativo foi observado em outros três artigos, ${ }^{25,30,86}$ gerando assim dúvidas sobre a qualidade científica dessas publicações.

A ausência de dois estudos com as mesmas terapias pode ser justificada pelo campo de ação proposto pela medicina antienvelhecimento, ${ }^{1}$ mas também impede análise comparativa e replicação do experimento, comprometendo o valor científico deles. Mesmo o envelhecimento cutâneo, objetivo encontrado em quatro desses artigos, ${ }^{14,28,30,67}$ foi avaliado com metodologias e terapias diferentes, impedindo análise qualitativa específica.

A categoria de recomendação "C" e o grau de evidência "II" refletem essas impropriedades metodológicas. Justifica-se também o baixo fator de impacto da maioria dessas publicações.

\section{CONCLUSÃO}

Tendo a presente revisão bibliográfica encontrado apenas nove trabalhos desenvolvidos exclusivamente em seres humanos ${ }^{14,17,25,28,30,64,67,86,109}$ e considerando a amplitude proposta como campo de ação da medicina antienvelhecimento, ${ }^{1}$ não há sólidas evidências científicas e/ou clínicas que justifiquem sua aplicabilidade na presente prática médica. 


\section{REFERÊNCIAS}

1. Fisher A, Morley JE. Antiaging medicine: the good, the bad, and the ugly. J Gerontol A Biol Sci Med Sci. 2002;57:M636-9.

2. Jadad AR, Moore RA, Carroll D, Jenkinson C, Reynolds DJ, Gavaghan DJ, et al. Assessing the quality of reports of randomized clinical trials: is blinding necessary? Control Clin Trials. 1996;17:1-12.

3. Phillips B, Ball C, Sackett D, Badenoch D, Straus S, Haynes B, et al. Oxford Centre for Evidence-based Medicine Levels of Evidence (May 2001). [acesso 1 Set 2008]. Disponível em: http://cebm.jr2.ox.ac.uk .

4. Journal Citation Report - 2003 Science Edition. [acesso 11 Set 2008]. Disponível em: http:/www.research .hbi.ir/impact/Journal\%20Citation\%20Report\%20\%202003.htm.

5. Son BK, Akishita M. Vascular calcification and antiaging. Clin Calcium. 2008;18:912-7.

6. Chanchal D, Swarnlata S. Novel approaches in herbal cosmetics. J Cosmet Dermatol. 2008;7:89-95.

7. Rattan SI. Principles and practice of hormetic treatment of aging and age-related diseases. Hum Exp Toxicol. 2008;27:151-4.

8. Bruce S. Complementary effects of topical antiaging treatments in conjunction with aesthetic procedures. J Drugs Dermatol. 2008;7:s23-7.

9. Bruce S. Cosmeceuticals for the attenuation of extrinsic and intrinsic dermal aging. J Drugs Dermatol. 2008; 7:s17-22.

10. Zhang Y, Dong XL, Leung PC, Che CT, Wong MS. Fructus ligustri lucidi extract improves calcium balance and modulates the calciotropic hormone level and vitamin D-dependent gene expression in aged ovariectomized rats. Menopause. 2008;15:558-65.

11. Enns LC, Wiley JC, Ladiges WC. Clinical relevance of transgenic mouse models for aging research. Crit Rev Eukaryot Gene Expr. 2008;18:81-91.

12. Marzetti E, Lawler JM, Hiona A, Manini T, Seo AY, Leeuwenburgh C. Modulation of age-induced apoptotic signaling and cellular remodeling by exercise and calorie restriction in skeletal muscle. Free Radic Biol Med. 2008;44:160-8.

13. Rajendrasozhan S, Yang SR, Kinnula VL, Rahman I. SIRT1, an antiinflammatory and antiaging protein, is decreased in lungs of patients with chronic obstructive pulmonary disease. Am J Respir Crit Care Med. 2008; 177:861-70.

14. Akhtar N, Yazan Y. Formulation and in-vivo evaluation of a cosmetic multiple emulsion containing vitamin $\mathrm{C}$ and wheat protein. Pak J Pharm Sci. 2008;21:45-50.

15. Chen CD, Podvin S, Gillespie E, Leeman SE, Abraham CR. Insulin stimulates the cleavage and release of the extracellular domain of Klotho by ADAM10 and ADAM17. Proc Natl Acad Sci USA. 2007;104:19796-801.

16. Talbourdet S, Sadick NS, Lazou K, Bonnet-Duquennoy M, Kurfurst R, Neveu M, et al. Modulation of gene expression as a new skin anti-aging strategy. $\mathrm{J}$ Drugs Dermatol 2007;6: S25-33.

17. Benito-Ruiz J, Raigosa JM, Manzano-Surroca M, Salvador L. Male chest enhancement: pectoral implants. Aesthetic Plast Surg. 2008;32:101-4.

18. Sultana N, Lee NH. Antielastase and free radical scavenging activities of compounds from the stems of Cornus kousa. Phytother Res. 2007;21:1171-6.

19. Luo D, Lin XF, Min W, Ma QH, Gu N, Jin SL, et al. Photoprotection by tocopherol submicron emulsion against UV-mediated damage in HaCaT cells. Methods Find Exp Clin Pharmacol. 2007;29:185-9.

20. Katsiki M, Chondrogianni N, Chinou I, Rivett AJ, Gonos ES. The olive constituent oleuropein exhibits proteasome stimulatory properties in vitro and confers life span extension of human embryonic fibroblasts. Rejuvenation Res. 2007;10:157-72.

21. Miller NL, Fulmer BR. Injection, ligation and transplantation: the search for the glandular fountain of youth. J Urol. 2007;177:2000-5.

22. Davis T, Wyllie FS, Rokicki MJ, Bagley MC, Kipling D. The role of cellular senescence in Werner syndrome: toward therapeutic intervention in human premature aging. Ann N Y Acad Sci. 2007;1100:455-69.

23. Briden E, Jacobsen E, Johnson C. Combining superficial glycolic acid (alpha-hydroxy acid) peels with microdermabrasion to maximize treatment results and patient satisfaction. Cutis. 2007;79:13-6.

24. Effron C, Briden ME, Green BA. Enhancing cosmetic outcomes by combining superficial glycolic acid (alpha-hydroxy acid) peels with nonablative lasers, intense pulsed light, and trichloroacetic acid peels. Cutis. 2007;79:4-8.

25. Shameem R, Latif SA. Healthy aged persons had a better adrenocortical function. Mymensingh Med $\mathrm{J}$. 2007; 16:61-3.

26. Haruna Y, Kashihara N, Satoh M, Tomita N, Namikoshi $\mathrm{T}$, Sasaki T, et al. Amelioration of progressive renal injury by genetic manipulation of Klotho gene. Proc Natl Acad Sci USA. 2007;104: 2331-6.

27. Kamel NS, Gammack J, Cepeda O, Flaherty JH. Antioxidants and hormones as antiaging therapies: high hopes, disappointing results. Cleve Clin J Med. 2006;73:1049-56,1058.

28. Metcalf S, Crowson AN, Naylor M, Haque R, Cornelison R. Imiquimod as an antiaging agent. $J$ Am Acad Dermatol. 2007;56:422-5.

29. Kreyden OP. Antiaging - a scientific topic or just a social trend? J Cosmet Dermatol. 2005;4:228-9.

30. Fournier N, Fritz K, Mordon S. Use of nonthermal blue (405- to 420-nm) and near-infrared light (850- to 900$\mathrm{nm})$ dual-wavelength system in combination with glycolic acid peels and topical vitamin $\mathrm{C}$ for skin photorejuvenation. Dermatol Surg. 2006;32:1140-6.

31. Kang SK, Kim KS, Kim SI, Chung KH, Lee IS, Kim CH. Immunosuppressive activity of deer antler extracts of Cervus korean TEMMINCK var. mantchuricus Swinhoe, on type II collagen-induced arthritis. In Vitro Cell Dev Biol Anim. 2006;42:100-7.

32. Arck PC, Overall R, Spatz K, Liezman C, Handjiski B, Klapp BF, et al. Towards a "free radical theory of graying": melanocyte apoptosis in the aging human hair follicle is an indicator of oxidative stress induced 
tissue damage. FASEB J. 2006;20:1567-9.

33. Fahy GM, Wowk B, Wu J. Cryopreservation of complex systems: the missing link in the regenerative medicine supply chain. Rejuvenation Res. 2006;9:279-91.

34. Calabrese V, Maines MD. Antiaging medicine: antioxidants and aging. Antioxid Redox Signal. 2006;8:362-4.

35. Zhang D, Zhang JJ. [Effect of Coeloglossum. viride var. bracteatum extract on oxidation injury in sub-acute senescent model mice]. Zhongguo Yi Xue Ke Xue Yuan Xue Bao. 2005; 27:729-33.

36. Micans $P$. The need for anti-aging medicine: the challenges faced to incorporate preventative medicine into the clinic and into society. Ann N Y Acad Sci. 2005; 1057: 545-62.

37. Oktem G, Uslu S, Vatansever SH, Aktug H, Yurtseven ME, Uysal A. Evaluation of the relationship between inducible nitric oxide synthase (iNOS) activity and effects of melatonin in experimental osteoporosis in the rat. Surg Radiol Anat. 2006;28:157-62.

38. Assimopoulou NA, Sinakos Z, Papageorgiou VP. Radical scavenging activity of Crocus sativus L. extract and its bioactive constituents. Phytother Res. 2005; 19:997-1000.

39. Perls TT, Reisman NR, Olshansky SJ. Provision or distribution of growth hormone for "antiaging": clinical and legal issues. JAMA. 2005;294:2086-90.

40. Oxenkrug G. Antioxidant effects of N-acetylserotonin: possible mechanisms and clinical implications. Ann N Y Acad Sci. 2005; 1053: 334-47.

41. Draelos ZD. Topical and oral estrogens revisited for antiaging purposes. Fertil Steril. 2005;84:291-2.

42. Hazzard WR. The conflict between biogerontology and antiaging medicine--do geriatricians have a dog in this fight? J Am Geriatr Soc. 2005;53:1434-5.

43. Whitehouse PJ, Juengst ET. Antiaging medicine and mild cognitive impairment: practice and policy issues for geriatrics. J Am Geriatr Soc. 2005;53:1417-22.

44. Grossman T. Latest advances in antiaging medicine. Keio J Med. 2005;54:85-94.

45. Farris PK. Topical vitamin C: a useful agent for treating photoaging and other dermatologic conditions. Dermatol Surg. 2005;31:814-7.

46. Cooper R, Morre DJ, Morre DM. Medicinal benefits of green tea: Part I. Review of noncancer health benefits. J Altern Complement Med. 2005;11:521-8.

47. Lee TK, Johnke RM, Allison RR, O'Brien KF, Dobbs LJ Jr. Radioprotective potential of ginseng. Mutagenesis. 2005;20:237-43.

48. Erol A. PPARalpha activators may be good candidates as antiaging agents. Med Hypotheses. 2005;65:35-8.

49. Assimopoulou AN, Papageorgiou VP. Radical scavenging activity of Alkanna tinctoria root extracts and their main constituents, hydroxynaphtho quinones. Phytother Res. 2005;19: 141-7.

50. Matsakas A, Diel P. The growth factor myostatin, a key regulator in skeletal muscle growth and homeostasis. Int J Sports Med. 2005;6:83-9.

51. Stock GB. The pitfalls of planning for demographic change. Ann N Y Acad Sci. 2004;1019:546-51.
52. Riga D, Riga S, Schneider F. Regenerative medicine: Antagonic-Stress therapy in distress and aging. I. Preclinical synthesis--2003. Ann N Y Acad Sci. 2004;1019:396-400.

53. Ukraintseva SV, Arbeev KG, Michalsky AI, Yashin AI. Antiaging treatments have been legally prescribed for approximately thirty years. Ann N Y Acad Sci. 2004;1019:64-9.

54. Fisher AL, Hill R. Ethical and legal issues in antiaging medicine. Clin Geriatr Med. 2004;20:361-82.

55. Banks WA, Farr SA. Antiaging methods and medicines for the memory. Clin Geriatr Med. 2004;20:317-28.

56. Hajjar RR. Cancer in the elderly: is it preventable? Clin Geriatr Med. 2004;20:293-316.

57. Brown-Borg HM. Antiaging supplement holds promise to halt age-related cognitive deterioration. Exp Biol Med (Maywood). 2004;229:367-8.

58. Navarro A. Mitochondrial enzyme activities as biochemical markers of aging Mol Aspects Med. 2004;25:37-48.

59. Briden ME. Alpha-hydroxyacid chemical peeling agents: case studies and rationale for safe and effective use. Cutis. 2004;73:18-24.

60. Carrillo-Vico A, Calvo JR, Abreu P, Lardone PJ, GarciaMaurino S, Reiter RJ, et al. Evidence of melatonin synthesis by human lymphocytes and its physiological significance: possible role as intracrine, autocrine, and/or paracrine substance. FASEB J. 2004;18:537-9.

61. Lomnitski L, Bergman M, Nyska A, Ben Shaul V, Grossman S. Composition, efficacy, and safety of spinach extracts. Nutr Cancer. 2003;46:222-31.

62. Juliet PA, Balasubramaniam D, Balasubramaniam $\mathrm{N}$, Panneerselvam C. Carnitine: a neuromodulator in aged rats. J Gerontol A Biol Sci Med Sci. 2003;58:970-4.

63. Aburjai T, Natsheh FM. Plants used in cosmetics. Phytother Res. 2003;17:987-1000.

64. Yang CC, Chen JC, Chen GW, Chen YS, Chung JG. Effects of Shao-Fu-Zhu-Yu-Tang on motility of human sperm. Am J Chin Med. 2003;31:573-9.

65. Russo A, Izzo AA, Borrelli F, Renis M, Vanella A. Free radical scavenging capacity and protective effect of Bacopa monniera L. on DNA damage. Phytother Res. 2003;17:870-5.

66. Bergamini E. In response to: "Is there an antiaging medicine?" J Gerontol A Biol Sci Med Sci. 2003;58:483.

67. Bauza E, Dal Farra C, Berghi A, Oberto G, Peyronel D, Domloge N. Date palm kernel extract exhibits antiaging properties and significantly reduces skin wrinkles. Int J Tissue React. 2002;24:131-6.

68. Juengst ET, Binstock RH, Mehlman MJ, Post SG. Aging. Antiaging research and the need for public dialogue. Science. 2003;299:1323.

69. Xing H, Hu X, Liu H, Li Y, Chen Y. [Study on DNA oxidative damage of $\mathrm{O} 3$ aging model in mice]. Hua $\mathrm{Xi}$ Yi Ke Da Xue Xue Bao. 2001;32:229-31.

70. Li K, Liu M, Wei X. Study of xixin, duzhong and mixture to the antioxidant system of D-galactose-induced senile mice. Zhong Yao Cai 2000;23:161-3.

71. Shimokawa I, Fukuyama T, Yanagihara-Outa K, Tomita M, Komatsu T, Higami Y, et al. Effects of caloric 
restriction on gene expression in the arcuate nucleus. Neurobiol Aging 2003;24:117-23.

72. Altwein JE, Keuler FU. [Prevention and complementary medicine in aging]. Urologe A. 2002;41:318-24.

73. Butler RN, Fossel M, Harman SM, Heward CB, Olshansky SJ, Perls TT, et al. Is there an antiaging medicine? J Gerontol A Biol Sci Med Sci. 2002;57:B333-8.

74. Olshansky SJ, Hayflick L, Carnes BA. Position statement on human aging. J Gerontol A Biol Sci Med Sci. 2002;57:B292-7.

75. Schneider RH, Alexander CN, Salerno JW, Robinson DK Jr, Fields JZ, Nidich SI. Disease prevention and health promotion in the aging with a traditional system of natural medicine: Maharishi Vedic Medicine. J Aging Health. 2002; 14:57-78.

76. Roth GS, Ingram DK, Lane MA. Caloric restriction in primates and relevance to humans. Ann N Y Acad Sci. 2001;928:305-15.

77. Shin KH, Lim SS, Lee SH, Lee YS, Cho SY. Antioxidant and immunostimulating activities of the fruiting bodies of Paecilomyces japonica, a new type of Cordyceps sp. Ann N Y Acad Sci. 2001;928: 261-73.

78. Knoll J. Antiaging compounds: (-)deprenyl (selegeline) and (-) 1-(benzofuran-2-yl)-2-propylaminopentane, [(-)BPAP], a selective highly potent enhancer of the impulse propagation mediated release of catecholamine and serotonin in the brain. CNS Drug Rev. 2001;7:317-45.

79. Bartke A, Coschigano K, Kopchick J, Chandrashekar V, Mattison J, Kinney B, et al. Genes that prolong life: relationships of growth hormone and growth to aging and life span. J Gerontol A Biol Sci Med Sci. 2001;56:B340-9.

80. Vojta CL, Fraga PD, Forciea MA, Lavizzo-Mourey R. Antiaging therapy: an overview. Hosp Pract (Minneap). 2001;36:43-9.

81. Russo A, Izzo AA, Cardile V, Borrelli F, Vanella A. Indian medicinal plants as antiradicals and DNA cleavage protectors. Phytomedicine. 2001;8:125-32.

82. Borek C. Antioxidant health effects of aged garlic extract. J Nutr. 2001;131:1010S-5S.

83. Farris PK. Office dispensing: a responsible approach. Semin Cutan Med Surg. 2000;19: 195-200.

84. Cui X, Dai XG, Li WB, Zhang BL, Fang YZ. Effects of LuDuo-Wei capsules on superoxide dismutase activity and contents of malondialdehyde and lipofuscin in the brain of the housefly. Am J Chin Med. 2000;28:259-62.

85. Calabrese V, Scapagnini G, Catalano C, Dinotta F, Geraci D, Morganti P. Biochemical studies of a natural antioxidant isolated from rosemary and its application in cosmetic dermatology. Int $J$ Tissue React. $2000 ; 22: 5-13$

86. Liu J, Wang S, Liu H, Zhang C, Yang L, Zhang J. The immunomarker of aging and the modulatory effect of Chinese medicinal herbs on the dysfunction of lymphocytes in the elderly. J Environ Pathol Toxicol Oncol. 2000; 19:153-8.

87. Shimokawa I, Yanagihara K, Higami Y, Okimoto T,
Tomita M, Ikeda T, et al. Effects of aging and dietary restriction on mRNA levels of receptors for growth hormone-releasing hormone and somatostatin in the rat pituitary. J Gerontol A Biol Sci Med Sci. 2000;55:B274-9.

88. Vazquez B, Ortiz C, San Roman J, Plasencia MA, LopezBravo A. Hydrophilic polymers derived from vitamin $\mathrm{E}$. J Biomater Appl. 2000;14:367-88.

89. Shimokawa I, Higami Y. A role for leptin in the antiaging action of dietary restriction: a hypothesis. Aging (Milano). 1999;11:380-2.

90. Calabrese V, Scapagnini G, Randazzo SD, Randazzo G, Catalano C, Geraci G, et al. Oxidative stress and antioxidants at skin biosurface: a novel antioxidant from lemon oil capable of inhibiting oxidative damage to the skin. Drugs Exp Clin Res. 1999;25:281-7.

91. Cui X, Dai XG, Li WB, Zhang BL, Fang YZ. Effects of luduo-wei capsule on prolonging life span of housefly and Drosophila melanogaster. Am J Chin Med. 1999;27: 407-13.

92. Calabrese V, Randazzo SD, Catalano C, Rizza V. Biochemical studies on a novel antioxidant from lemon oil and its biotechnological application in cosmetic dermatology. Drugs Exp Clin Res .1999;25:219-25.

93. Boisnic S, Branchet-Gumila MC, Le Charpentier Y, Segard C. Repair of UVA-induced elastic fiber and collagen damage by $0.05 \%$ retinaldehyde cream in an ex vivo human skin model. Dermatology. 1999; 199Suppl1:43-8.

94. Calabrese V, Randazzo SD, Morganti PG, Rizza V. An ex vivo biochemical model to study the antioxidant clinical properties of cosmetic products in human antiaging skin care. Drugs Exp Clin Res. 1999;25:43-9.

95. Manyam BV. Dementia in Ayurveda. J Altern Complement Med. 1999;5:81-8.

96. Misra R. Modern drug development from traditional medicinal plants using radioligand receptor-binding assays. Med Res Rev. 1998;18:383-402.

97. Khalsa DS. Integrated medicine and the prevention and reversal of memory loss. Altern Ther Health Med. 1998; 4:38-43.

98. Novelli M, Masiello P, Bombara M, Bergamini E. Protein glycation in the aging male Sprague-Dawley rat: effects of antiaging diet restrictions. J Gerontol A Biol Sci Med Sci. 1998;53:B94-101.

99. Ma YX, Yue Z, Wang ZS, Chen SQ, Liang ZJ, Zhang JG, et al. Physiological basis for long life span. Mech Ageing Dev. 1997;98:47-55.

100. Ma YX, Zhu Y, Wang CF, Wang ZS, Chen SY, Shen MH, et al. The aging retarding effect of 'Long-Life CiLi'. Mech Ageing Dev. 1997;96:171-80.

101. Li JQ, Li ZK, Duan H, Zhang JT. [Effect of age and gin senoside Rg1 on nitric oxide content and nitric oxide synthase activity of cerebral cortex in rats]. Yao Xue Xue Bao. 1997; 32:251-4.

102. Li JQ, Zhang JT. [Effects of age and ginsenoside RG1 on membrane fluidity of cortical cells in rats]. Yao Xue Xue Bao. 1997;32:23-7.

103. Nishiyama N, Moriguchi T, Saito H. Beneficial effects of aged garlic extract on learning and memory 
impairment in the senescence-accelerated mouse. Exp Gerontol. 1997;32:149-60.

104. Isoyama S. Age-related changes before and after imposition of hemodynamic stress in the mammalian heart. Life Sci. 1996;58:1601-14.

105. Zhao Y, Wang X, Kawai M, Liu J, Liu M, Mori A. Antioxidant activity of Chinese ant extract preparations. Acta Med Okayama 1995;49:275-9.

106. Liu M, Zhang J. Effects of ginsenoside Rb1 and Rg1 on synaptosomal free calcium level, ATPase and calmodulin in rat hippocampus. Chin Med J (Engl). 1995; 108:544-7.

107. Nose Y. 5th WAA Congress therapeutic artificial organs, 10 years after. Artif Organs 1995;19:204-10.

108. Ortmeyer HK, Bodkin NL, Hansen BC. Chronic calorie restriction alters glycogen metabolism in rhesus mon keys. Obes Res. 1994;2:549-55.

109. Predescu V, Riga D, Riga S, Turlea J, Barbat IM, BotezatAntonescu L. Antagonic-stress. A new treatment in gerontopsychiatry and for a healthy productive life. Ann N Y Acad Sci. 1994; 717:315-31.

110. Randerath K, Li D, Nath R, Randerath E. Exogenous and endogenous DNA modifications as monitored by 32P-postlabeling: relationships to cancer and aging. Exp Gerontol. 1992;27:533-49.

111. Chung MH, Kasai H, Nishimura S, Yu BP. Protection of DNA damage by dietary restriction. Free Radic Biol Med. 1992;12:523-5.

112. Regelson W, Franson R. Phospholipase A2 as a "death trigger" in the aging process. The use of PLA2 inhibitors as antiaging substances. Ann N Y Acad Sci. 1991;621:262-76.

113. Liu J, Edamatsu R, Kabuto H, Mori A. Antioxidant action of guilingji in the brain of rats with $\mathrm{FeCl} 3$-induced epilepsy. Free Radic Biol Med. 1990;9:451-4.
114. Frontiers in bioscience: Impact Factor of Journals in the Order of Impact Factor. [acesso 1 Set 2008]. Disponível em: www.bioscience.org/ services/impact15.htm

115. Sociedade Brasileira de Profissionais em Pesquisa Clínica - SBPPC. Manual sobre Pesquisa Clínica voltado para o paciente. [acesso 13 Set 2008]. Disponível em: http://www.sbppc.org.br

116. Brasil - Ministério da Saúde - Conselho Nacional de Saúde. Resolução 196/96 - Diretrizes e normas regulamentadoras de pesquisas envolvendo seres humanos. [acesso 13 Set 2008]. Disponível em: http://conselho.saude.gov.br/comissao/conep/ resolucao.html.

117. Brasil - Ministério da Saúde - Conselho Nacional de Saúde. Resolução 251/97 - Normas de pesquisa com novos fármacos, medicamentos, vacinas e testes diagnósticos envolvendo seres humanos. [acesso 13 Set 2008]. Disponível em: http://conselho.saude. gov.br/comissao/conep/resolucao.html .

118. Brasil - Ministério da Saúde - Agência Nacional de Vigilância Sanitária. Resolução RDC n³9 sobre regulamento para a realização de pesquisa clínica. [acesso 13 Set 2008]. Disponível em: http://e-legis. anvisa.gov.br/leisref/public/showAct.php?id=31279 .

\footnotetext{
ENDEREÇO PARA CORRESPONDÊNCIA / MAILING ADDRESS:

Milton Luiz Gorzoni

Hospital Geriátrico e de Convalescentes Dom

Pedro II

Avenida Guapira, 2674

02265002 São Paulo SP

Tel.:/Fax: 1121761204 / 5589-9408

E-mail: bdp.dirtec@santacasasp.org.br
}

Como citar este arquivo/How to cite this article: Gorzoni ML, Pires SL. Há evidências científicas na medicina antienvelhecimento? An Bras Dermatol. 2010;85(1):57-64. 